\title{
Structural design of independent metering control systems
}

\author{
A. Sitte, J. Weber \\ Institute of Fluid Power, Technical University Dresden, Dresden/Germany \\ E-mail: sitte@ifd.mw.tu-dresden.de, weber@ifd.mw.tu-dresden.de
}

\begin{abstract}
This paper regards to design and examination of electrohydraulic control systems with separate metering edges for mobile applications. Although the idea of individual metering has existed for some time, this promising technology has yet to be applied in field. One reason for the missing prevalence is the lack of understanding regarding the complex control requirements. Furthermore, expected additional expenses regarding components such as valves and transducers hinder the acceptance. This work outlines a systematic analysis of possible circuit concepts. The obtained solution space contains a large number of elements that must be evaluated based on functional and economical criteria. Therefore, it is advantageous to divide the control system into its functional fractions. The clear distinction of the functional fractions enables a systematization of the different drive and control tasks, which then can be combined freely. The selection of appropriate control signals can be supported by the usage of a multivariable system modeling approach. Physical interactions, inherent to electrohydraulic systems, have a strong impact on the behavior of the control loops. By analyzing the coupling elements, conclusions regarding control concepts and structures can be drawn.
\end{abstract}

Keywords: electrohydraulic control systems, separate metering, MIMO, valve structures

\section{Introduction}

In mobile machinery it is very common that multiple hydraulic drives are supplied by one single pump. The actuators shall be operated independently and simultaneously. The hydraulic control system has to distribute the hydraulic power among the actuators depending on their flow and pressure requirements. Process forces mostly appear in both positive and negative direction. Negative load forces in direction of movement occur for example during lowering of loads or when decelerating moving masses. This typically leads to an overrunning load and thus an uncontrolled acceleration of the hydraulic motor. Moreover negative pressures may occur, since the pump flow is not sufficient for the filling of the cylinder. In order to prevent damage resulting from cavitation phenomena usually suction valves are used. Furthermore overcentre valves are utilized to prevent uncontrolled movement. The throttling cross section is varied as a function of the inlet pressure and thus limits the actuator's velocity. Disadvantages of this setup are increased power consumption, especially at partial loads, and the dynamic behavior of the drive system, which tends to oscillate in an unfavorable manner. Modern systems meet most of the requirements concerning controllability but system limits are increasingly reached in terms of efficiency and dynamics. A reasonable and promising way to overcome this issue is the use of hydraulic systems with independent metering edges [4], [24] and [10]. By doing so, the opening cross section of the meter-out edges can be adapted to the load situation and can be manipulated independently from the inlet. By influencing the pressure level, regeneration and recuperation modes become possible. A separate control of the tank edge also opens up for minimal pressure losses at all working conditions. Cavitation during pulling load situations can be avoided without extensive control edge design. Furthermore, the distributed valve arrangement allows the use of decentralized control architectures.

\section{State of the Art}

A natural approach to derive systems with independent metering properties is to add one control input in order to control an additional state variable. As a result, a hardware layout with two 3/3-valves is obtained. These circuit variants are preferred in [11], [4], [27], and [5]. Pressure sensors are used for controlling the main valve [14], [15]. The design of those systems differs considerably. [14] uses the idea of classic load sensing with primary pressure compensation. Although a load-independent actuator movement is implemented, at least two additional pressure transducers are needed. Analogue solutions are published in numerous patents and scientific contributions [16], [17], [18], [19], and [28]. Industrial applications by Eaton [1] and Wessel [8] have come to attention as well. In [20] a valve arrangement with independently controlled metering edges 
and the use of secondary pressure compensators is proposed. Thus, a typical flow divider setup is provided. In case of saturation of the pump flow, the flow will still be distributed proportionally in correlation to the valves opening area ratios. The resulting component effort is affected negatively, since two additional pressure compensators are needed. In addition to system solutions utilizing 3/3-valves, especially concepts with $2 / 2$-valves are in the scope of research. These valve types are often simpler and more cost effective. Configurations with 2/2-valves are introduced in [9], [25], and [26] [21], [22], [12]. Eriksson and Palmberg present a system without pressure sensors [6], [23]. Each main valve is equipped with a pressure compensator. The load conditions are determined by measuring the position of the pressure compensators. Among others, Linjama and Villenius deal with stability and control issues [10].

\section{Derivation of circuit structures for control systems with independent metering edges}

Based on today's control systems, the demands in individual metering control can be described as follows:

- Lowering the energy consumption by reducing the losses at the tank edges

- Simplifying the design of control edges in comparison to conventional systems

- Simple control strategies for operating the individual metering elements and thus achieving at least as good dynamic performance as existing control systems

- Reduction of components expense (solenoids, valves, sensors) compared to previously known system solutions with separate control edges

- Control on the basis of the latest electrohydraulic load-sensing systems

- Load compensation for parallel operation of several actuators

- Simple implementation of float functionality

- Simple implementation of circuits to exploit recuperation and regeneration potentials

The approach for finding new solutions comprises a systematic analysis of possible circuit concepts.

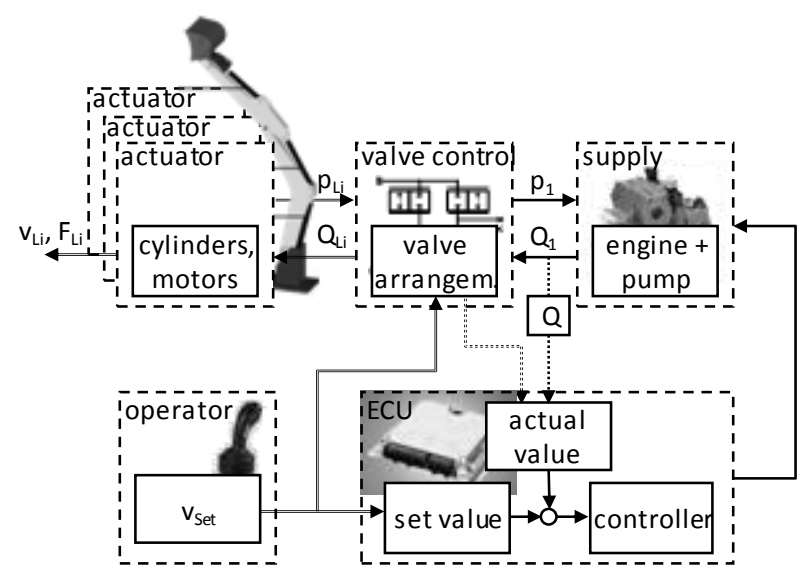

Figure 1: General electrohydraulic structure of working hydraulics for mobile machinery
The obtained solution space contains a large number of elements that must be evaluated based on functional and economical criteria. Therefore, it is advantageous to divide the control systems into its functional areas, see Figure 1. For each of these areas a number of solutions are possible. The clear distinction of the functional areas (operator, supply, valve control system, ECU and actuator) enables a systematization of the different drive and control tasks, which can be combined freely.

\subsection{Main valve architectures}

In order to develop circuit principles two design aspects are considered. The first one concerns the valve type combinations while the second one regards the arrangement of elements. Serial arrangements of two proportional metering edges result in redundant structures, which are unfavorable in terms of component expense. Likewise, parallel assemblies of a proportional edge and a switching edge are not applicable, since the control feature of the proportional edge is short circuited, see Figure 2.

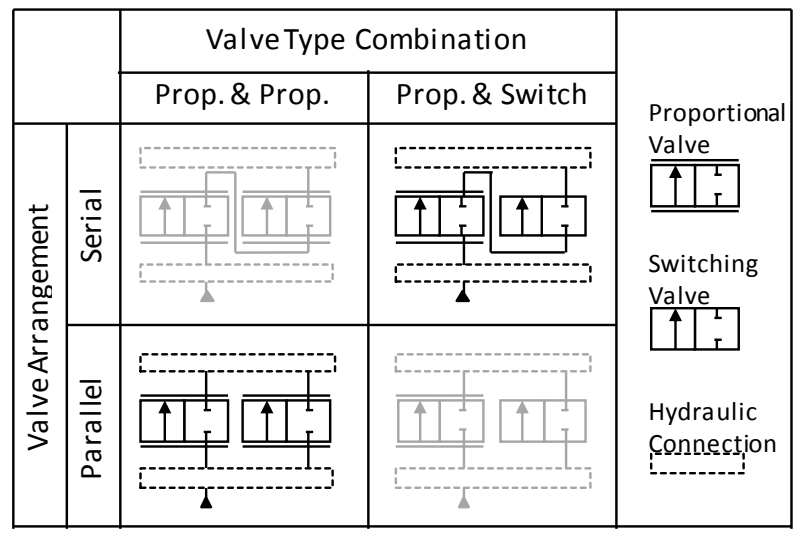

Figure 2: Methodology of determining fundamental circuit principles

The essential requirement of an individual metering layout for a single consumer port corresponds to a 3/3-valve-type design. Under this premise, only circuit principles with at least 3/3-valve functionality are suitable. To determine whether a circuit principle meets the necessary functioning condition, mathematical formulations are used to calculate the equivalent valve functionality. On the basis of an electrical circuit analogy, parallel elements may be added, whereas elements connectec in series are multiplied. A parallel and a serial arrangement of two 2/2-way proportional metering edges serves as an example. Each of the elements contains one independent connection per switch position and two switching positions including null. Adding the number of independent connections i.e. the number of switching positions, which corresponds to a parallel arrangement, results in a 3/3-valve functionality. For a serial arrangement, it is necessary to multiply the characteristic numbers. As expected, the result shows a 2/2valve functionality.

The systematic de-/coupling of the mechanical constraints starting from 4/3-valve design results in different hardware layouts. In general, the circuit principles can be classified in 
symmetric and asymmetric circuits. Symmetric circuits only use one valve type for implementation of basic working functions, whereas asymmetric circuits use at least two different proportional valve types. The functionally and mechanically coupled metering cross section can be allocated either at the working ports $\left(\mathrm{p}_{\mathrm{A}}\right.$ or $\left.\mathrm{p}_{\mathrm{B}}\right)$ or at the supply ports $\left(\mathrm{p}_{0}\right.$ or $\mathrm{p}_{\mathrm{T}}$ ) see Figure 3 .

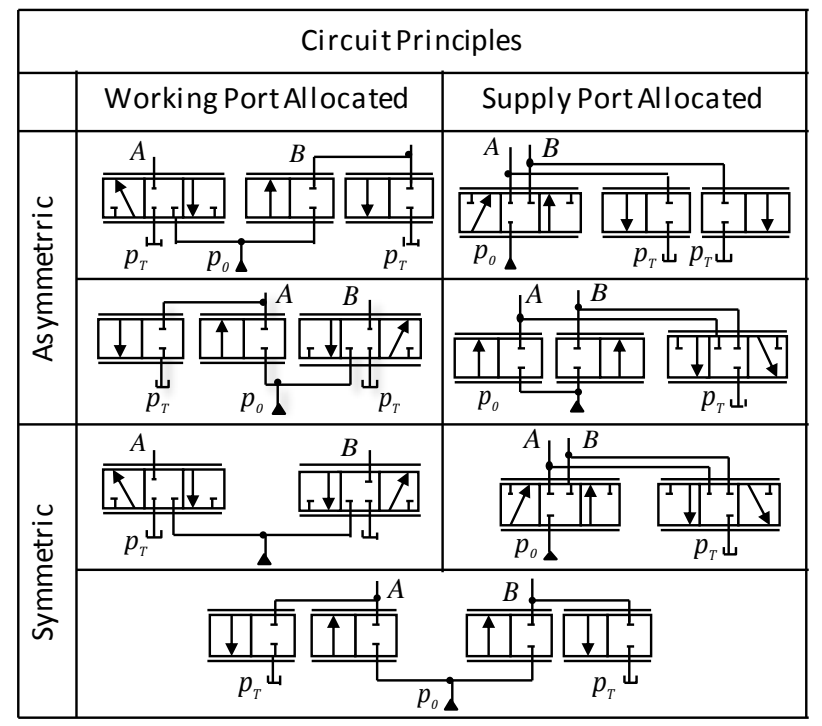

Figure 3: Fundamental circuit principles with parallel arrangement of proportional valve elements

The basic circuit principles can be compared based on criteria like the number of necessary valves or flexibility of arrangement. Also the type of solenoid, whether proportional or switching type, influences the choice. An adoption of $2 / 2$-valves enables the usage of poppet type valves. There are several benefits in using poppet type valves, for example the much simpler valve design or nearly leakage-free characteristic. Furthermore, it is possible to mount these valves directly to the actuator. Enhanced dynamics can be achieved because hose and pipe volumina between valve and cylinder are kept small. Instead of connecting each individual consumer to the supply, pressure and tank line can be connected from one actuator to another. With the introduced combination methodology, several thousand possible valve arrangements are conceivable. But only a handful of them are of relevance for application.

\subsection{Individual flow control}

To attain load independent actuator motion, a constant flow at varying operating pressures over the control valves is necessary. This is accomplished by individual flow control. A circuitry implementation can be performed mechanically by individual pressure compensators or by means of electronics with appropriate sensors, see Figure 4. In contrast to sensors, mechanical solutions react directly and rapidly on disturbance variables in the system. However, system performance is influenced because damping might be reduced. The combination of the different possible solutions for the different sub-divisions gives an overview of the results in their entirety.

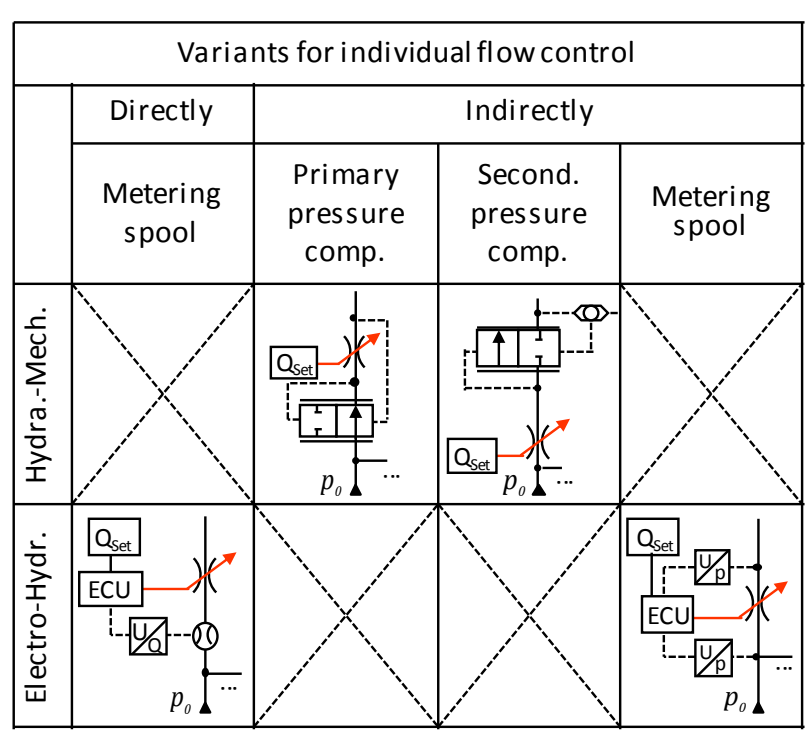

Figure 4: Variants of individual flow control

A conventional pressure compensated hydromechanical load sensing-system serves as a basis of comparison. The result of the evaluation is a ranked list of structures. Some of the solutions are presented in the following.

\subsection{Determining circuitry concepts}

In literature mostly symmetric and parallel arrangements of proportional valves as shown in Figure 3 are suggested, since this reflects the intuitive approach of separating the metering edges. A further aspect that correlates with the separation-thought is the disunion of metering and directing function. A circuit principle that implements such a task has been introduced in Figure 2 by a serial arrangement of a proportional and switching valve. A low-pressure drop and applicability of a simple and therefore low priced actuating solenoid are only some of the benefits of switching valves. By systematically combining different valve types and control circuit principles, a solution space is identified. In Figure 5 an extract from the resulting set of possible solutions is presented. These novel structures are characterized by an economical use of components. In comparison to known structures, the component effort in terms of complexity and number is reduced. The proposed system layouts utilize two 2/2-proportional valves for metering and two 3/2-switching valves for flow directing functionality. Depending on the configuration, one or two pressure compensators are required. In that way pre- or post compensation can be implemented. To fully use the potentials concealed in the control concept, additional pressure sensors or displacement sensors are necessary. Along with dynamic and functional issues, there are safety issues to consider. 


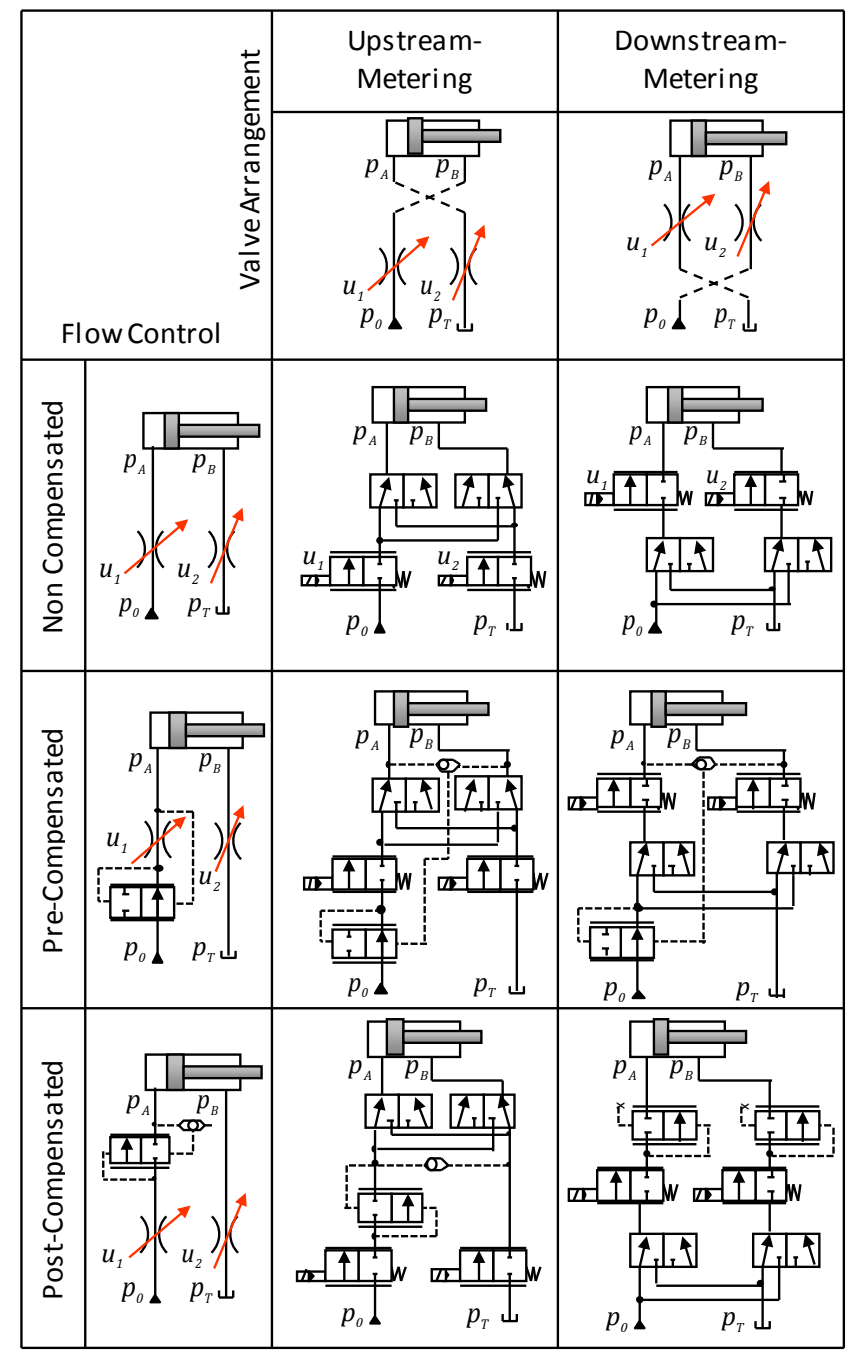

Figure 5: Extract of combination matrix containing novel structures

\section{Modeling and simulation of systems with independent metering edges}

In this chapter, general mathematical models of the individual metering systems are derived. Linearized models are used for analysis of the systems behavior. The investigations are exemplarily conducted on the noncompensated and the primary-compensated system layout, see Figure 5.

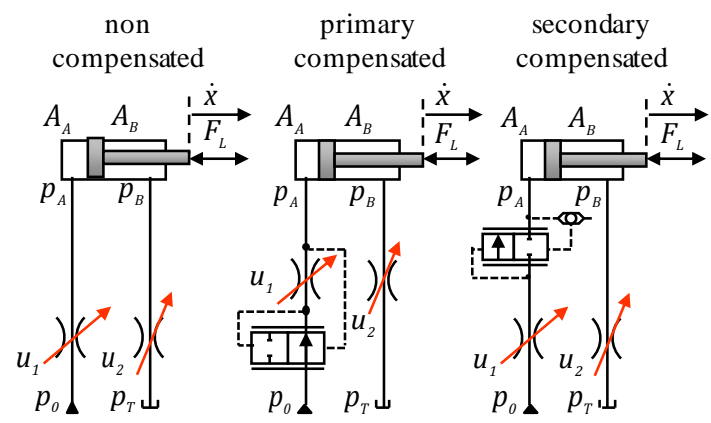

Figure 6: Simplified system layouts
To simplify analysis, the presence of only one actuator is assumed. The pump supply can be modeled using a transfer function with second order dynamics. The spool dynamics of the individual pressure compensator (IPC) as well as the dynamics of the cylinder can be described with a second order transfer function. However, the spool dynamics of the IPC can be modeled statically, since the corresponding inertias are small in comparison to the dynamics of the actuator line. The pressure build-up in hydraulic capacities is of first order. Experimental data of the static flow characteristic of the control valve are plotted in Figure 7 along with simulation results of the non-linear model.

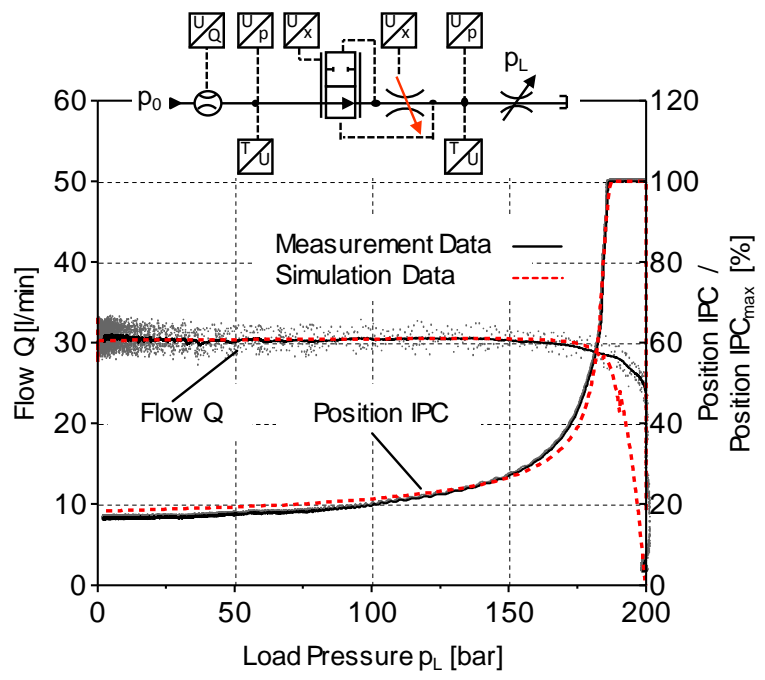

Figure 7: Static flow characteristic, stroke of individual pressure compensator of control valve

The model has been verified in the same manner for different operating points. In general the model showed a good quality match for the static behavior. However, there are deviations concerning certain dynamic phenomena, like excess flows, which are well known effects in two-way flow control valves. Since these deviations result in slight differences in the overall system performance, they may be neglected for further analysis.

\subsection{Generalized linear models}

Linearizing a non-linear model always leads to a compromise, because the resulting system is only valid locally around a specific operating-point. One of the possibilities to overcome this problem is provided by an operating-point tracing. All operating-point-dependent terms are being updated within each simulation run, to obtain model validity.

A minimal set of equations is needed to describe the systems behavior. The linearized flow equation is used to describe turbulent flow through a cross section

$$
\begin{aligned}
& Q_{A}=K_{Q u 1} \cdot u_{1}+K_{Q p 1} \cdot\left(p_{0}-p_{A}\right) \\
& Q_{B}=K_{Q u 2} \cdot u_{2}+K_{Q p 2} \cdot\left(p_{B}-p_{T}\right)
\end{aligned}
$$

where $K_{Q u i}$ is the constant flow coefficient and $p_{i}$ are the system pressures. The continuity equation in hydraulic lines 
describes the pressure build-up with the corresponding hydraulic capacitances $K^{\prime} / V_{i}$

$$
\begin{aligned}
& Q_{A}-A_{A} \cdot \dot{x}=\frac{K^{\prime}}{V_{A}} \cdot \dot{p}_{A} \\
& Q_{B}-A_{B} \cdot \dot{x}=\frac{K^{\prime}}{V_{B}} \cdot \dot{p}_{B}
\end{aligned}
$$

In combination with the motion equation of a differential cylinder, it represents the dynamics of the system

$$
m \cdot \ddot{x}=p_{A} \cdot A_{A}-p_{B} \cdot A_{B}-F_{L}
$$

Leakage effects, which mainly results in damping, is neglected. The resulting system block diagram is shown in Figure 8 .

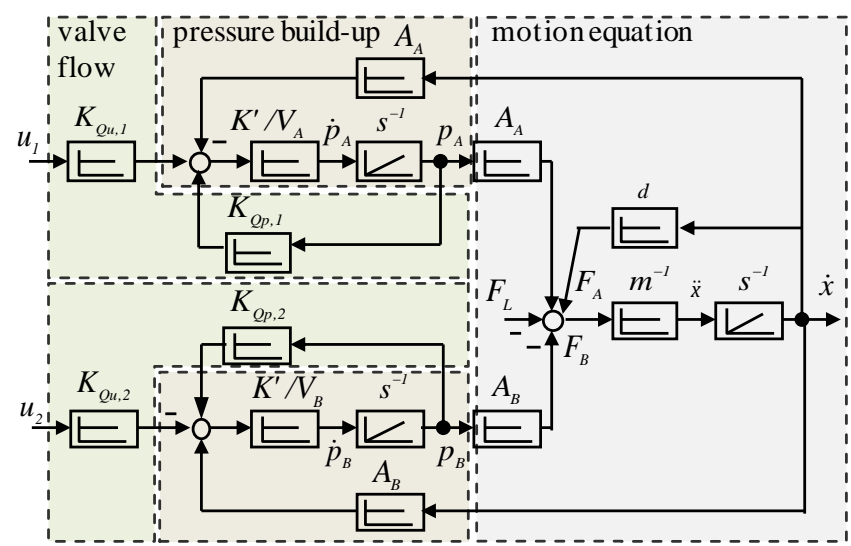

Figure 8: Linearized and simplified model of noncompensated hydraulic control system

The extension of the system with an individual pressure compensator in a pre-compensation layout is shown in Figure 9. As noted before, the spool dynamics of the IPC can be modeled statically, since the dynamic is much higher than the cylinder dynamics.

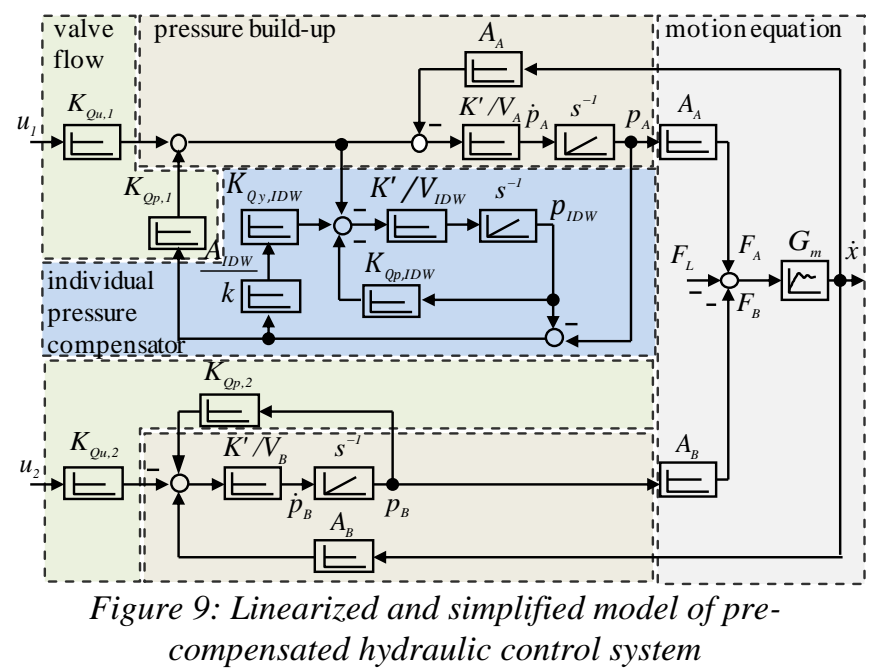

The static motion equation and pressure built-up are noted in Eq. (6) and Eq. (7)

$$
\begin{aligned}
& y_{I D W}=\frac{A_{I D W}}{k} \cdot\left(p_{I D W}-p_{A}\right) \\
& Q_{I D W}-Q_{A}=\frac{K^{\prime}}{V_{I D W}} \cdot \dot{p}_{I D W}
\end{aligned}
$$

The individual pressure compensator controls the pressure difference across the measuring orifice by throttling the cross sectional flow. In case of load or speed variations, the IPC changes its position in a closed loop characteristic, keeping the pressure difference $\Delta p=\left(p_{I D W}-p_{A}\right)$ constant. The resulting flow through the control valve can be written as

$$
\begin{aligned}
& Q_{I D W}=K_{Q y, I D W} \cdot y_{I D W}+K_{Q p, I D W} \cdot\left(p_{0}-p_{I D W}\right) \\
& Q_{A}=K_{Q u 1} \cdot u_{1}+K_{Q p 1} \cdot\left(p_{I D W}-p_{A}\right)
\end{aligned}
$$

It is obvious that the resulting systems with more than one input can no longer be considered as single input systems. In fact, it was the goal all along to control more than one output value, by introducing another input value. In order to continue using linear control design methods, a transformation of the system defining equations becomes necessary. The multivariable system analysis is presented in the following section.

\subsection{Multivariable system approach}

For the solution of analysis and design tasks, it is sometimes important to know which subsystems the multivariable system consists of. Structural information can be used to recognize internal feedbacks in the control system or to identify interactions between several control loops. Two important model forms are the p-canonical and v-canonical representation of multivariable systems, see Figure 10. It is a major objective of structured representation to distinguish main-couplings and cross-couplings. Therefore, these structures are mainly utilized for systems with the same number of input and output variables.

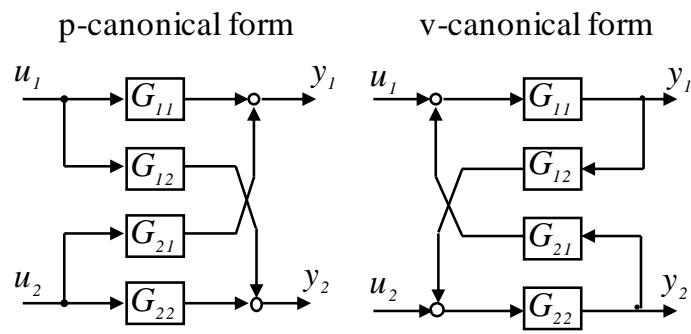

Figure 10: General description of multiple-input multipleoutput systems (MIMO)

Multivariable systems can be classified in positively and negatively coupled systems. In case of equal signs (positive or negative), there is a positive coupling. Diverse signs indicate a negative coupling. These types of systems are generally associated with less damping. The influence of the coupling elements becomes particularly clear if the respective other transmission element represents a transfer function parallel to the main transfer function.

The p-canonical structure can be considered as the "natural" structure, which is derived out of the matrix representation 
of the system. However, it is not "natural" in the sense of the physical structure. The individual transmission elements $G_{i i}$ do not describe separate physical processes, but only cover different signal couplings within the same system. This fact becomes particularly obvious when certain system parameters occur in several transfer functions simultaneously.

The resulting p-canonical representation of the multivariable system is shown in Figure 11.
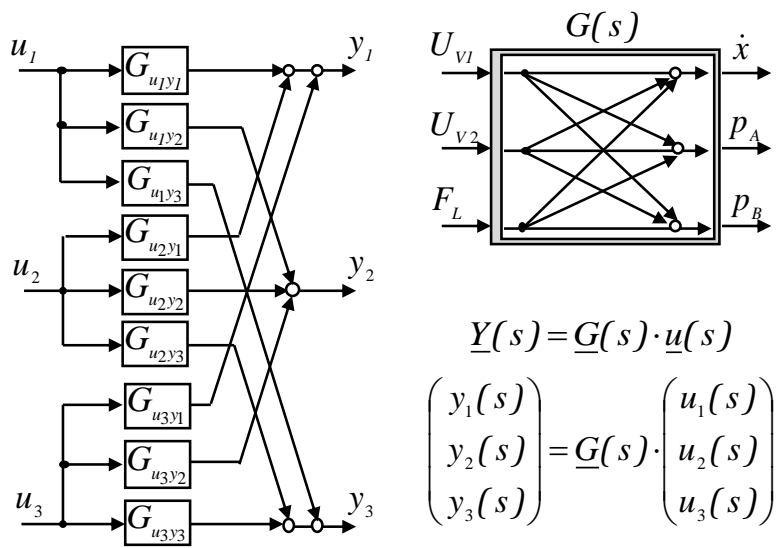

$\underline{Y}(s)=\underline{G}(s) \cdot \underline{u}(s)$

$$
\left(\begin{array}{l}
y_{1}(s) \\
y_{2}(s) \\
y_{3}(s)
\end{array}\right)=\underline{G}(s) \cdot\left(\begin{array}{l}
u_{1}(s) \\
u_{2}(s) \\
u_{3}(s)
\end{array}\right)
$$

Figure 11: Representation of considered MIMO system with independent metering edges

The matrix containing the transfer functions can be written as

$$
\left(\begin{array}{l}
y_{1} \\
y_{2} \\
y_{3}
\end{array}\right)=\left(\frac{1}{N(s)}\right) \cdot\left(\begin{array}{lll}
G_{u_{1}, y_{1}} & G_{u_{2}, y_{1}} & G_{u_{3}, y_{1}} \\
G_{u_{1}, y_{2}} & G_{u_{2}, y_{2}} & G_{u_{3}, y_{2}} \\
G_{u_{1}, y_{3}} & G_{u_{2}, y_{3}} & G_{u_{3}, y_{3}}
\end{array}\right)\left(\begin{array}{l}
u_{1} \\
u_{2} \\
u_{3}
\end{array}\right)
$$

The aforementioned equation neglects operation point dependent terms, which result from calculation of the Taylor series. The equations (11) to (22) evaluate the transfer functions.

$$
\begin{aligned}
& G_{B 1}=K_{Q p 1}+\frac{V_{A}}{K_{O ̈ l}^{\prime}} \cdot s \\
& G_{B 2}=K_{Q p 2}+\frac{V_{B}}{K_{O ̈ l}^{\prime}} \cdot s \\
& N(s)=m s \cdot G_{B 1} \cdot G_{B 2}+A_{A}^{2} \cdot G_{B 2}+A_{B}^{2} \cdot G_{B 1} \\
& G_{u_{1}, y_{1}}(s) \cdot N(s)=G_{B 2} \cdot K_{Q u_{1}} \cdot A_{A} \\
& G_{u_{2}, y_{1}}(s) \cdot N(s)=G_{B 1} \cdot K_{Q u_{2}} \cdot A_{B} \\
& G_{u_{3}, y_{1}}(s) \cdot N(s)=-G_{B 1} \cdot G_{B 2} \\
& G_{u_{1}, y_{2}}(s) \cdot N(s)=K_{Q u 1}\left(G_{B 2} \cdot m \cdot s+A_{B}^{2}\right) \\
& G_{u_{2}, y_{2}}(s) \cdot N(s)=-K_{Q u 2} \cdot A_{A} \cdot A_{B} \\
& G_{u_{3}, y_{2}}(s) \cdot N(s)=G_{B 2} \cdot A_{A}
\end{aligned}
$$

$$
\begin{aligned}
& G_{u_{1}, y_{3}}(s) \cdot N(s)=K_{Q u 1} \cdot A_{A} \cdot A_{B} \\
& G_{u_{2}, y_{3}}(s) \cdot N(s)=-K_{Q u 2}\left(G_{B 1} \cdot m \cdot s+A_{A}^{2}\right) \\
& G_{u_{3}, y_{1}}(s) \cdot N(s)=-G_{B 1} \cdot A_{B}
\end{aligned}
$$

The transfer functions $G_{i i}$ for a variation of cylinder stroke $x=($ Min ... Max $)$, which mainly results in different capacities, are plotted in Figure 12. There is a peak in all the transfer functions for the chosen operating point. This resonance is the hydraulic resonance frequency of the spring-mass system.

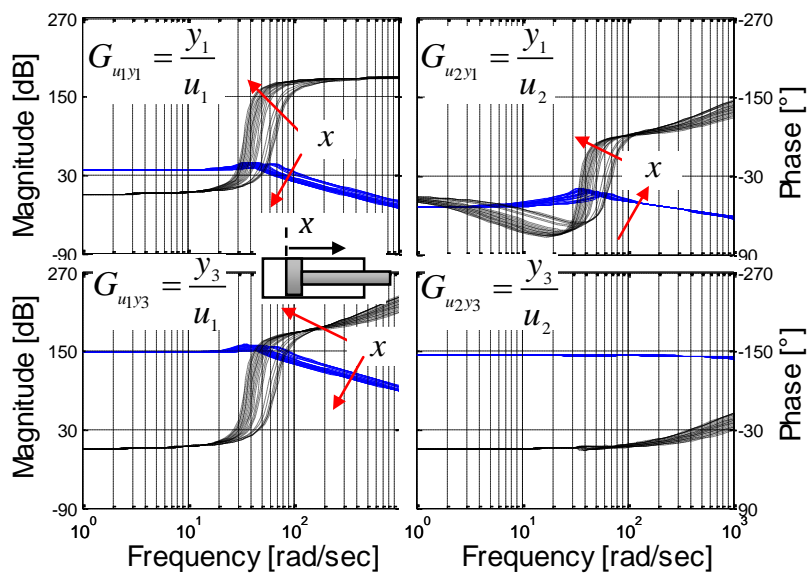

Figure 12: Open loop transfer function of multiple-input multiple-output system (MIMO) without IPC

The Eigenfrequency of the system shifts towards lower values, since the piston side volume increases. As there is no substantial resistance at the meter out edge, which would cause a pressure built up, the frequency of the system does not increase like it would in conventional hydraulic systems with one single spool. The corresponding pole-zero-map is plotted in Figure 13. For a decreasing signal at the meter-in valve $u_{l}=U_{V l}$ the poles tend to move towards the right complex half plane with decreasing flow across the main valve.

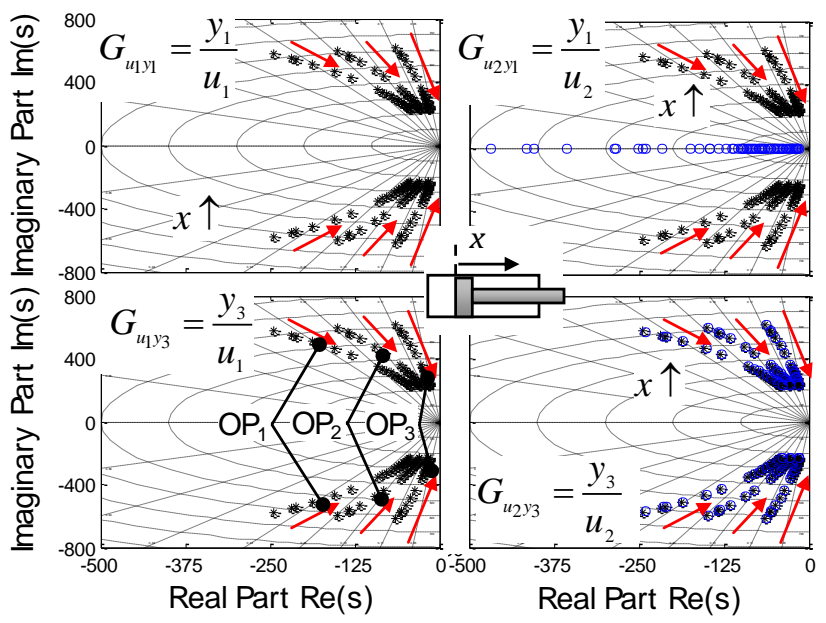

Figure 13: Pole zero map of open loop transfer function without IPC 
The arrows indicate the variation of the frequency along the piston stroke. Whereas the operating points $O P_{1}$ to $O P_{3}$ stand for different actuator velocities. The damping is mainly dependent on viscous friction and flow characteristic of the system. By introducing sensors to feed back state variable information, the dynamics of main-couplings as well as cross-couplings are affected. That is when stability issues have to be taken into account.

Analogous to the model description of the non-compensated system, the equations (23) to (28) are introduced to model a system with primary pressure compensator.

$$
\begin{aligned}
& G_{B 1}=\frac{K_{\ddot{O} l}}{V_{A}} \cdot\left(1+\frac{K_{Q p, 1} \cdot G_{3}}{1-G_{c I D W} \cdot G_{3}}\right) \cdot s+ \\
& K_{Q p, 1} \cdot\left(1+\cdot \frac{1}{1-G_{I D W} \cdot G_{B 3}}\right) \\
& G_{B 2}=K_{Q p 2}+\frac{V_{B}}{K_{\ddot{O} l}^{\prime}} \cdot s \\
& G_{B 3}=\frac{k}{A_{I D W} \cdot K_{Q y, I D W}} \\
& G_{I D W}=\frac{V_{I D W}}{K_{O ̈ l}^{\prime}} \cdot s+K_{Q p, I D W} \\
& F_{1}=\frac{1}{1+\frac{K_{Q p, 1} \cdot G_{B 3}}{1-G_{c I D W} \cdot G_{B 3}}} \\
& N(s)=m s \cdot G_{B 1} \cdot G_{B 2}+\frac{A_{A}^{2}}{F_{1}} \cdot G_{B 2}+A_{B}^{2} \cdot G_{B 1}
\end{aligned}
$$

The entries of the matrix $\underline{G}(s)$, which represent the transfer functions of the subsystems, are equivalent to the equations (14) to (22). The corresponding dynamics are plotted in Figure 14.

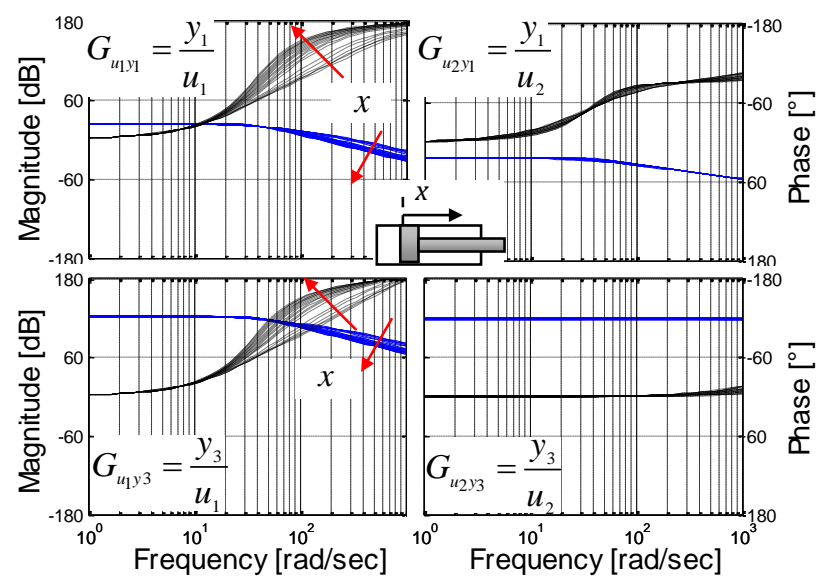

Figure 14: Open loop transfer function of multiple-input multiple-output system (MIMO) with IPC

Analyzing the systems pole-zero-map, see Figure 15, it is apparent, that varying the cylinder stroke $x$ has huge impact on the locations of the roots. The damping is significantly reduced with increasing piston stroke. The angular frequency however is not affected as severely as in the noncompensated system.

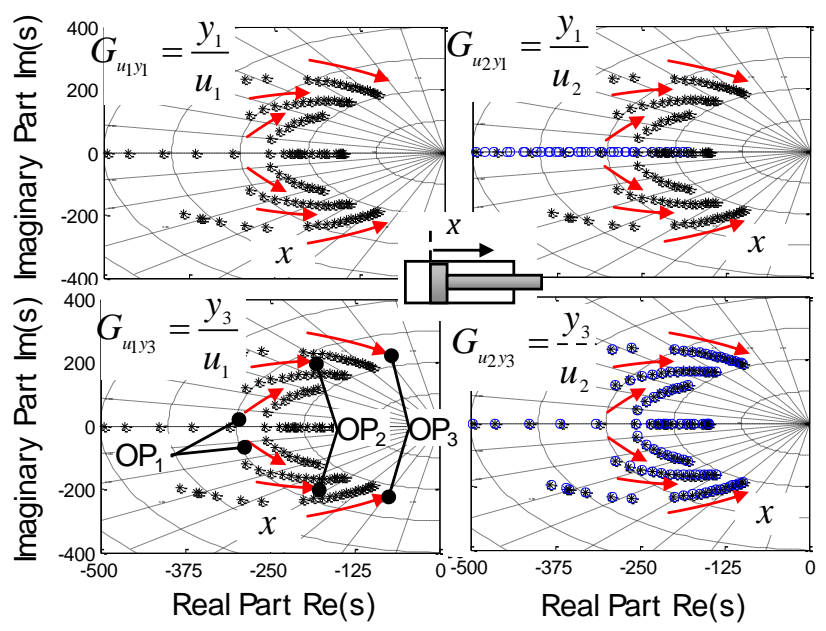

Figure 15: Pole zero map of open loop transfer function with IPC

The cross talk between input variable $u_{1}$ and $u_{2}$ is decreased. This is because the pressure difference across the measurement orifice is kept constant by the pressure compensator. While the pressure compensator's position is located in the control range, load variations do not influence the flow through the control valve.

\subsection{Multivariable system analysis}

The selection of appropriate control signals is a question of structural nature, since it is not only dependent on specific system parameters but on fundamental transmission properties of the controlled system. Accordingly, the structural properties of the controlled system are the primary factors in determining appropriate control signals. The decision on a certain control structure is significantly affected by the properties of dynamic systems. These properties are as follows:

- In order to stabilize an unstable system path, the control signals and controlled variables must be selected in a way that the unstable eigenvalues of the system become controllable and observable.

- The selected control signals and the controlled variables must not be linearly dependent. This condition is violated when the matrix $\mathrm{B}$ or $\mathrm{C}$ of the state space model do not meet a certain rank criterion.

- There must always exist at least as many control signals as controlled variables.

- All-pass behavior of the system path and the corresponding control signal complicates the control task.

- All-pass behavior may be eliminated by introducing additional control signals.

The preliminary decision, whether a multivariable controller is necessary or several single loop control circuits are 
sufficient mainly depends on the strength of the couplings. In the literature, various empirical coupling measures are proposed. The question, which remains, is the strength of the cross-coupling elements $G_{12}$ and $G_{21}$ on the behavior of the control loop. In particular, it is interesting to know the range of values these elements may reach without affecting the stability of the control loops. In the following section, an assessment of the couplings is made to come to a decision whether a multivariable control is required or not.

\subsection{Definition and analysis of coupling-factor}

To evaluate the couplings, a distinct assignment of input and output variables is necessary. Furthermore, a preliminary control structure must be assumed. Since the problem of input-output assignment is trivial for the system considered, following control structure is obtained, see Figure 16.

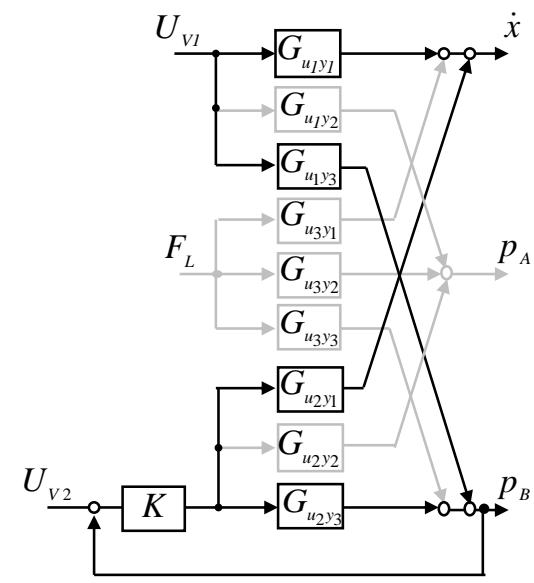

\section{Figure 16: Block Diagram of reference behavior of the controlled MIMO system}

The illustration above shows the familiar structure of the MIMO system, which has been extended with a pressure feedback on the meter-out side. The meter-in valve is still responsible for velocity control in an open loop manner. This would be a common and simple approach to control a system with independent metering edges.

The determinant of the feedback matrix $\underline{F(s)}$ is of major importance for the stability analysis. Internal couplings of the system may be evaluated by examining the crosscoupling-elements $G_{12}$ and $G_{21}$. Computing the determinant of $\underline{F}(s)$, one partial result is the so called coupling factor noted in equation (29)

$$
\kappa(G(s))=\left(\frac{G_{12}(s) \cdot G_{21}(s)}{G_{11}(s) \cdot G_{22}(s)}\right)
$$

If there were no cross-couplings, $G_{12}$ and $G_{21}$ would be equal to zero and two decoupled transmission lines would exist. The absolute value of the expression $\kappa(s)$ provides information on the strength of the couplings. If the absolute value of $\kappa(s)$ is much smaller than one, there is a low coupling. Any value close to or greater than one implies strong couplings. The coupling coefficient $\kappa(s)$ describes the strength with which the first control loop influences the transmission behavior of the second main control line. If $\kappa(s)$ is very small, the controller $K(s)$ may be designed only according to the behavior of the second main control path $p_{B}(s)=G_{u 2 y 3}(s) U_{V 2}(s)$. However, if $\kappa(s)$ is large the transmission behavior of the cross- and main-couplings influences the controller design. The term $\kappa(s)$ only states an empirical coupling-measure and therefore it only provides a guide value. The resulting couplings of the considered system layout are plotted in Figure 17. Displayed are two system layouts with- and without individual pressure compensator at two different load conditions.

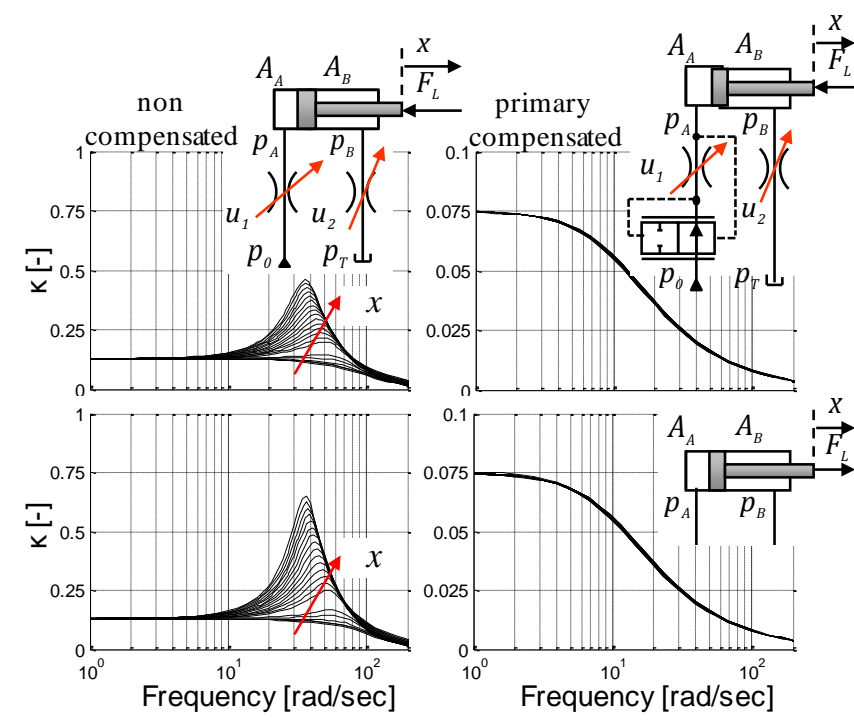

Figure 17: Couplings of the considered system with and without IPC

The top left diagram shows the gradient of the coupling factor, which has a maximum value of 0.5 . In the figure below, the equal variations are displayed in case of pulling load characteristics. The value of $\kappa(s)$ even increases. Since these values are relatively high, the system has to be considered a multivariable system. By introducing the individual pressure compensator to the system, the couplings between the main control lines are reduced significantly, see Figure 17 top-right. Since the coupling factor does not increase when pulling loads occur, a decentralized and therefore single input controller may be designed. A similar behavior can be observed when utilizing post-compensation. While the pressure compensator of the flow divider circuit is working within its control range, it shares likewise characteristics. Since it is one demand to implement simple and robust control methods, the use of individual pressure compensators appears to be desirable.

\section{Conclusion and outlook}

The paper started with a brief review on the state of the art for control systems and valve concepts with independent metering edges. In the following a systematic approach for determining circuitry principles was introduced. In the next paragraph a parametrised non-linear model of a noncompensated and a pre-compensated system have been presented. Some effects like rigid friction, leakage and spool dynamics have been neglected for the purpose of simplification. In order to obtain a canonical model structure 
of the multivariable input output system, a linearization has been conducted. The models of both system layouts have been applied to an analysis of the systems couplings. The decision on a certain control structure is significantly affected by the properties of dynamic systems, which can be described with the help of the system couplings. The analysis showed that utilizing mechanical pressure compensators is favourable in terms of the necessary control effort. System couplings are reduced over a wide range of operation. Based on that knowledge, a single input controller layout may be used in order to keep implementation and commissioning effort low.

Since the control system design is highly dependent on the application and the machine structure, it is necessary to take different machines and working functions into consideration. Relevant application areas are primarily in the field of working hydraulics of construction machinery. These machines cover a wide range of applications and load conditions. A comprehensive analysis of machines and their associated application spectrum has to be carried out to develop suitable control system solutions. In combination with an operating strategy this will be the next step to be investigated.

\section{Acknowledgements}

The work reported in this paper was performed within the project: "New Electrohydraulic Control Systems with Independent Metering Edges" funded by the DFG (German Research Society GZ: WE 4828/1-1). The permission for publication is gratefully acknowledged.

\section{Nomenclature}

\begin{tabular}{lll}
\hline Designation & Denotation & Unit \\
\hline$A_{i}$ & Cylinder area & {$\left[\mathrm{m}^{2}\right]$} \\
$d$ & Viscous friction coefficient & {$[\mathrm{Ns} / \mathrm{m}]$} \\
$E C U$ & Electronic control unit & {$[-]$} \\
$F_{L i}$ & Load force & {$[\mathrm{N}]$} \\
$G_{i i}$ & Transfer function & {$[-]$} \\
$G$ & Matrix of transfer functions & {$[-]$} \\
$K$ & Feedback gain & {$[-]$} \\
$k$ & Spring rate & {$[\mathrm{N} / \mathrm{m}]$} \\
$K$ & Effective bulk modulus & {$[\mathrm{bar}]$} \\
$K_{Q p, i}$ & Lin. flow-pressure coefficient & {$[1 / \mathrm{min} / \mathrm{bar}]$} \\
$K_{Q u, i}$ & Lin. flow coefficient & {$[1 / \mathrm{min} / \mathrm{mm}]$} \\
$\kappa$ & coupling coefficient & {$[-]$} \\
$p_{L i}$ & Pressure & {$[\mathrm{bar}]$} \\
$\dot{p}_{L i}$ & Pressure build-up & {$[\mathrm{bar} / \mathrm{s}]$} \\
$Q_{L i}$ & Flow & {$[1 / \mathrm{min}]$} \\
$s$ & Laplace operator & {$[-]$}
\end{tabular}

\begin{tabular}{lll}
$u_{i}$ & Input & {$[-]$} \\
$U_{V i}$ & Valve voltage & {$[\mathrm{V}]$} \\
$v_{L i}, \dot{x}$ & Velocity & {$[\mathrm{m} / \mathrm{s}]$} \\
$V_{i}$ & Volume & {$\left[\mathrm{m}^{3}\right]$} \\
$y_{i}$ & Output & {$[-]$} \\
\hline
\end{tabular}

\section{References}

[1] Lunze, J.,Regelungstechnik 2 Mehrgrößensysteme Digitale Regelung, Springer, 2005.

[2] Anon, Eaton Ultronics Management System, Brochure No. E-VLDI-MR003-E2 Eaton Cooperation, 2005.

[3] Djurovic, M., Energiesparende Antriebssysteme für die Arbeitshydraulik mobiler Arbeitsmaschinen „Elektrohydraulisches Flow Matching“ Dissertation TU Dresden, 2007.

[4] Eriksson, B.; Larson, J.; Palmberg J.-O Study on Individual Pressure Control in Energy Efficient Cylinder Drives, 4th FPNI-PhD Symposium, Sarasota USA, 2006.

[5] Elving, M; Palmberg, J.-O. Distributed Control of Fluid Power Actuator - A Load-Sensing Application of a Cylinder with Decoupled Chamber Pressure Control 5th Scandinavian International Conference on Fluid Power, Linköping, 1997.

[6] Eriksson, B; Rösth, M.; Palmberg, J.-O. A, High Energy Efficient Mobile Fluid Power System - Novel System Layout and Measurement, $6^{\text {th }}$ International Fluid Power Conference, Dresden, 2008.

[7] Finzel, R; Elektrohydraulische Steuerungssysteme für mobile Arbeitsmaschinen, Dissertation TU Dresden, 2010.

[8] Jongebloed, H.; Fees, G. Proportional Directional Valve with Autonomous Spools (PAS) for Mobile Applications, $6^{\text {th }}$ International Fluid Power Conference 6.IFK, Dresden, 2008.

[9] Jansson, A.; Palmberg, J.-O. Separate Controls of Meter-in and Meter-out Orfices in Mobile Hydraulic Systems, Intenational Off-Highway \& Power Plant Congress and Exposition, Milwaukee (USA), 1990.

[10]Linjama, M.; Huova, M.; Vilenius, M. Online Minimisation of Power Losses in Distributed Digital Hydraulic Valve Systems, $6^{\text {th }}$ International Fluid Power Conference 6. IFK, Dresden, 2008.

[11] Matilla, J., On Energy Efficient Motion Control of Hydraulic Manipulators, $\mathrm{PhD}$ Thesis, Tampere University of Technology, 2000. 
[12] Meilicke, D., Rough Terrain Lift Truck Efficiency and Control Improvement through Electronically Controlled Distributed Valves, CFP, Las Vegas (USA), 2008.

[13] German Patent DE 10200705403536A1, Energierückgewinnungs- und Wiedernutzungsverfahren für ein Hydrauliksystem, HUSCO International, 2008.

[14] United States Patent 5568759 Hydraulic circuit having Dual Electro-hydraulic Control Valves, Caterpillar Inc., 1996.

[15]European Patent EP 1710446A2, Hydraulische Steueranordnung und Steuerblock, Bosch Rexroth AG, 2006.

[16] United States Patent 005947140, System and method for controlling an independent metering valve, Caterpillar Inc., 1999.

[17] United States Patent 006976418, Hydraulic System with an actuator having independent meter-in and meter-out control, Caterpillar Inc., 2006.

[18]Europeans Patent 0809737, Electrohydraulic proportional control valve assemblies, Eaton Ultronics, 1996.

[19] International Patent WO 2007/015814, EH Metering valve with integral flow control, Caterpillar, 2007.

[20] International Patent WO 02/075162 A1, Control Valve, Bucher Hydraulics GmbH, 2002.

[21] United States Patent 002770046, Integrated valve assembly and computer control for a distributed hydraulic control system, Husco International Inc., 2007

[22]Pfaff, J.; Distributed Electro-hydraulic Systems for Telehandlers, 50th National Conference on Fluid Power, Las Vegas (USA), 2005.

[23] United States Patent 6073652, Pilot Solenoid Control Valve with Integral Pressure Sensing Transducer, Husco International Inc., 2000.

[24] Shenouda, A.; Quasi-Static Hydraulic Control Systems and Energy Savings Potential using Independent Metering Four-Valve Assembly Configuration, $\mathrm{PhD}$ studies, Georgia Institute of Technology, USA, 2006.

[25] Shenouda, A.; Book, W.; Selection of Operating Modes of a Multi-Functional Hydraulic Device, ASME International Mechanical Engineering Congress and Exposition, Orlando (USA), 2005.

[26] Tabor, K.; A Novel Method of Controlling a Hydraulic Actuator with four Valve Independent Metering using Load Feedback; SAE Commercial Vehicle Engineering Conference, Chicago (USA), 2005.
[27] Yuan, Q.; Lew, J.; Modelling and Control of Two Stage Twin Spool Servo-Valve for Energy-Saving, American Control Conference; Portland, USA, 2005.

[28]Zhang, J.; Pressure Compensated Independent Metering Valve for EH System; NCFP, Las Vegas (USA), 2008. 